\title{
Analysis on Marketing Strategies of Tencent Games-Take Honor of Kings as an Example
}

\author{
Dantong Shen ${ }^{1 *}$ \\ ${ }^{1}$ School of Management and Economics \\ The Chinese University of Hong Kong \\ Shenzhen, China
}

\begin{abstract}
This paper mainly focuses on the marketing strategies of Tencent Games. Tencent Games has the largest revenue all over the world. It is considered that the effective marketing strategies play important roles in global competition. Many researches have been focusing on this topic. In this article, strategies are summarized into four main points: product marketing, entertainment marketing, cross-border cooperation and intellectual property creation. Then the author conducts out a deep case study of Honor of Kings, introducing the most striking and latest strategies of the game. The game has cross-border cooperation with a cosmetics brand, idolizes the in-game characters and gets hold of festivals to launch virtual products. The experience of Tencent Games indicates that different strategies fit different periods and the developer should listen to the users and follow the trend.
\end{abstract}

\section{Introduction}

In the $21^{\text {st }}$ century, with the rapid popularization and innovation of electronic products, more and more video games have penetrated into people's lives. Games market is now promising and plentiful. At the same time, it is highly competitive.

Tencent is one of the most important names of mobile games industry. The company owns a great number of famous games including Player Unknown's Battlegrounds (PUBG), Honor of Kings, Fortnite, Cross Fire and other games. In the past decade, the annual online games revenue of Tencent has shown a steady and unstoppable growth. In 2019, Tencent's online games grossed 115 billion yuan - more than a ten-fold increase compared to 2010[1]. According to the Global Games Market Report of Newzoo, Tencent has remained as the world's top game publisher by revenue in 2018, followed by Sony and Microsoft.

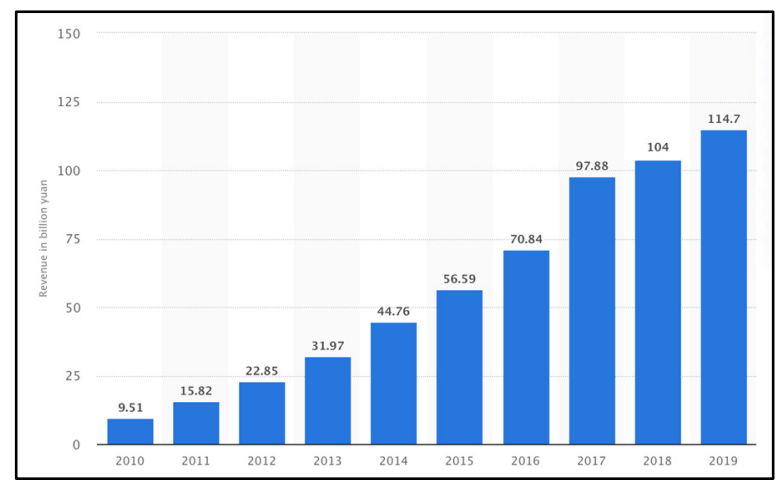

Fig1. Annual online games revenue of Tencent in the past ten years

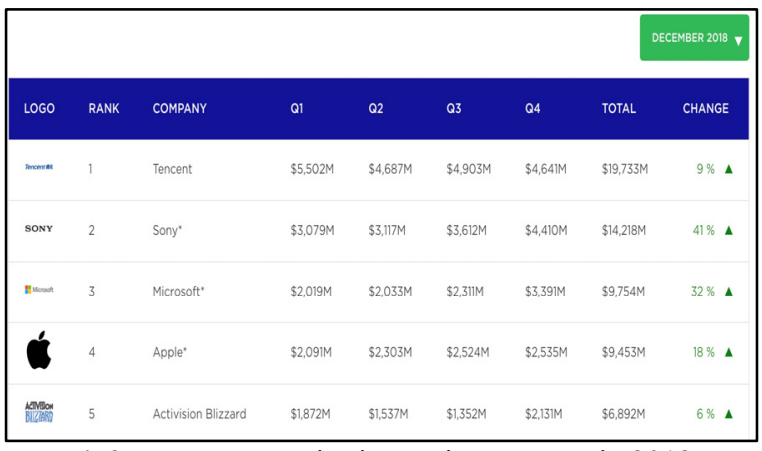

Fig2. Top 5 companies in gaming revenue in 2018 .

The game market, especially mobile game market, is still expanding fast today, thanks to the rapid technology innovation in smart phones. With mobile phones, people may click on game apps at any fragmented time when they are on buses or during class breaks. It is a crucial task for game companies to promote their games, thus people will subconsciously choose their games in an instant. Additionally, the companies need to think about how they can remain popularity and user loyalty from countless choices.

Marketing strategies play key roles in this situation. Tencent Games, as an industry leader, surely has some distinctive and remarkable methods to make the products stand out. This paper will dig deeply into the marketing strategies of Tencent Games. The significance of the paper are as follows.

1) Summarize the excellent marketing strategies of Tencent Games and use them as reference for other companies or fields.

2) To see whether Tencent Games can develop more marketing tools to have a further promotion of the games. 
3) To see how marketing on games brings the whole company more revenue.

The research on this topic is practical and concrete. It is conducted in a qualitative way. The most typical marketing strategies of Tencent Games are first summarized and explained with examples. Then, there is a case study of the success of Honor of Kings, which is one of the most profitable and successful game IP of Tencent in recent years.

\section{Literature review}

There is a considerable amount of literature on the video game's marketing strategies. Some researchers have already collected the promotion behaviors of game companies. In Marketing strategy analysis of mobile games-Taking King of Glory Games as an example, Li listed out several main ideas of game marketing, including integrated marketing, crossover marketing, brand marketing and precision marketing[2]. A disadvantage of her paper is that it only expressed in general term and did not go into much details.

$\mathrm{Li}$ pointed out that integrated marketing refers to integration of individual marketing tools into a whole, which can achieve synergies. The mobile game industry will make full use of huge network resources in promotion, combine online promotion with offline activities, and integrate various resources to serve the promotion of the same game[2]. In his analysis of marketing strategies of mobile game brands, Mingyang Ma indicated that through corporation with live broadcast platform, the game can have more exposure to the public and game users can have a certain understanding of the basic operation of the game[3]. Ma also raised an idea that some games had urban and campus competition for attracting attention[3]. Setting up prizes for game winners could not only motivate the players, but also improve the reputation of brand in public.

In terms of crossover marketing, $\mathrm{Li}$ gave some examples[2]. One example is that users can get free mobile data to play by downloading the game, and another example refers to the interaction with film and entertainment, including film and television implantation and celebrity endorsement. $\mathrm{Li}$ also stated that precision marketing, which made use of new media means to push marketing information to specific target consumer groups, is now prevailing and quite effective[2].

The above papers give us a general idea of how game companies may promote and spread their games. Unfortunately, some literature fails to combine viewpoints with concrete examples, namely games products. Instead of focusing on the product strategy of one specific company, the researchers simply generalize the strategies, which may be shared by other companies. Many questions remain unanswered such as in which situation should a specific strategy be used? Should the promotion methods be adjusted at different stages of the product life cycle? What kind of methods enable a game to stand out in the market? In short, the effect of marketing strategies has not been shown or determined in these papers.

Several studies have focused on the promotion of specific game products.

Honor of Kings has been a hot topic among the researchers. Yuxi Qiao concluded six main marketing strategies of this game in her research on innovative marketing strategies[4]. The strategies are shown below.

1) Use opinion leaders to expand game's popularity.

2) Network interaction (with QQ, weChat) for

increasing user stickiness

3) Launch a pattern for fragmented time

4) Use Star Effect to increase popularity

5) Launch multifarious skins and property in game at low cost

6) Bring out derivative products and culture

Her illustration is quite clear and concrete. However, some of the points are not really relevant to the game marketing. Additionally, since the paper was published two years ago, the latest and most innovative strategies are not included.

Jing Zhang and Wuqiang Tan studied on brand communication strategy of League of Legends, another famous game of Tencent[5]. There are posters and theme Internet cafes which are offline. As for online media, game videos, novice teaching videos and competition videos can attract the attention of players. We Media communication complements with star effect marketing. In the creative mining of culture, television and animation industry are booming.

Bingyu Zou and Xinji Chen investigated on a game called Identity $V$, a game of NetEase[6]. They illustrated that the game used cross-boundary cooperation to raise attention. Last April, Identity $V$ cooperated with Strawberry Music Festival to play game background music at the festival. Last November, Identity $V$ collaborated with animation "ITO run's second surprise collection" written by the famous Japanese terrorist master ITO runji and launched a new skin of characters. In addition, the game and Shanghai Happy Valley together launch the map of "Moon River Park".

These studies are more specific and practical. However, because of the rapidly changing games market, some strategies may be out of date. In the study of Honor of Kings in this paper, the author tries to cover all the latest and innovative marketing methods. The existing studies also seldom talk about the ultimate effect of strategies as the researchers are objective, which means that they stand on the sidelines and has not thought as a game player or potential customer. This paper tries to turn off these flaws.

\section{Analysis of Marketing Strategies}

In this part, the marketing strategies of Tencent Games are classified into four main aspects. The analysis covers the most typical and successful strategies, which distinguished Tencent Games from its competitors' product. Some points are obtained from speeches on Tencent Game Developers Conference (TGDC), a conference on which the developers share their ideas of game development and marketization.

Tencent has an innovation team called TGideas. According to its manager Ruofan Li, TGideas commits to help the products to communicate and connect with users 
effectively through creative design means and highquality derivative contents, and thus accomplish business value of products[7]. This team is the hero in marketing games. Its creative design marketing can run through the whole life cycle of a game.

\subsection{Four classification}

\subsubsection{Product marketing}

Product marketing is based on content of the game, including game playing, art images and the world view of the game. The main idea of product marketing is to decorate and package these things to make the game more attractive. The LOGO, ICON, official website and trailer together structure the image and temperament of the game product. This strategy is usually used before or at the first stage of its launch. This strategy is explicit and common. The posting is usually through mandatory advertising, a kind of one-way transmission of information which may not be so effective.

However, Tencent does a good job in product differentiation. TGideas design a unique set of visual symbols so that each game can show its own features. When TGideas are to design a creative plan for Monument Valley II, a game of ultimate beauty, the team devises a QR code which contains the beauty element in the game. The result of the design is particularly good since the simple code has brought 130,000 downloads directly. Tencent proves that even the basic marketing tool can break out a big hit when it is used properly.

\subsubsection{Entertainment marketing}

Entertainment marketing is oriented at exposure and spread of the game by using some attractive tools. It refers to celebrity endorsement, vital events, jokes and some other communication materials.

Entertainment marketing is usually used after the game comes out. The celebrities can improve the popularity of the game in a short time and attract the fans. Tencent uses this strategy in a new way and always looks for stars who fit the game's attributes. The company once even created a figure of the star in a game called The King of Fighters: Destiny. This strategy was novel at that time and refreshed the user's visual impression of the game.

\subsubsection{Cross-border cooperation}

As mentioned in the literature review, cross-border cooperation is a widely used strategy, mostly used after the game enters the mature stage. This strategy has two striking effects: bring users more experience outside the game product and thus provide a feeling of freshness; achieve cross penetration of users on both sides.

Here the author briefly introduces the case of $Q Q$ Speed. The game group is mainly composed of high school or college students. Tencent aimed to bring positive value guidance to young people. The innovation team planned an activity with VANS, a high street brand since 1966. In the following two years, eight episodes of documentaries, focusing on skateboarding, music, graffiti and some other trends and cultures have been published, bringing a full range of content experience for game users. The documentaries enable young people in China to feel the lifestyles and values of young people from other parts of the world. The scenes in documentaries were also introduced into the game as tracks and maps for attraction. In a word, this case of cooperation not only promote the positive ideas and values but also maintain and enhance the heat of the game.

\subsubsection{Intellectual property creation}

For many players, playing a game is not only because of its core gameplay or image design, but also because of its world view architecture, wonderful story and characters. Based on the same theme, namely the intellectual property (IP), a game can be transformed into movies, comics or even theme parks. IP is the soul of a game.

The creation and design of intellectual property can expand the mature stage of a game and thus the lifetime of the game. Honor of Kings does quite well in this aspect (which will be discussed in next part).

The design of IP brings the characters closer to real world. A mature IP can feed the second-generation products and help the new product go online. Furthermore, when a game is updated to an IP, it becomes more profitable. The derivative products, the in-game characters, the related animation can all earn money for the game company.

\subsection{A Case Study of Honor of Kings}

This part focuses on a game called Honor of Kings. The game first launched in 2016 and swept across the globe in a short period. It grossed nearly $\$ 2$ billion in 2018, making it the highest grossing mobile game of the year[8]. Till now the game has been successfully built into a brand, which touches many fields. Obviously, the success of Honor of Kings depends on many factors, including its high quality and operability. In this part, only the contribution of promotion strategies is concerned.

The marketing methods of Honor of Kings are of all aspects, through the whole life cycle. From the launch of the game, Tencent is very focused on how active it is in the online community. Honor of Kings has a super topic, which is a huge group for the players to chat, on Weibo. Veteran players and star players will upload their game operation videos on Weibo or provide life broadcasts. At the same time, offline competitions are held every year. As an Esport, Honor of Kings even enter into colleges. Thanks to these promotions, Honor of Kings quickly occupied the market. Entertainment marketing plays a significant role in this process.

When the game is already known to the public, Tencent does a lot of work to bring more fresh feelings. Many of the latest strategies are unique since they are unusual in other games.

One sensational strategy is the cross-border cooperation between Honor of Kings and M.A.C, a 
cosmetic brand. In 2019, the two brands released five cobranded lipsticks, featuring the lip colors of five wellknown game characters. This collaboration was inspired by the players of Honor of Kings. The cosmetics brand observed the players attempting to find lipstick shades that perfectly match the lip colors of their beloved game characters; these players mentioned M.A.C a number of times. Moreover, the two brands share the same target audience, who are girls between the ages of 18 and 24 . These factors created a perfect opportunity for a crossindustry collaboration[9]. The marketing turned to be a big success. Over 14,000 pre-orders were placed and all five shades sold out across all sales channels within 24 hours of the launch.

Another wisdom decision is the idolization of the characters in the game. Instead of getting a popular star to endorse the game, which is the strategy in the early stage, Tencent use the hot characters in the game to promote. More than $80 \%$ of the characters in game are Chinese historical celebrities or heroes from excellent literature. In 2019, 5 most popular male characters were picked out to form a virtual idol group, which seemed attractive to girls. The group later published songs and shew on the TV programs. Tencent skillfully use the characters to strengthen the connection between players and the game. This strategy is a novel use of the entertainment marketing.

The game is also good at promotion on festivals. Players can purchase virtual products, such as skins for characters in the game. Honor of Kings launches products of different themes on different festivals, such as Valentine's Day and Spring Festival. For example, a bridal skin was introduced on a Valentine's Day. Since many of these products are limited in a short period, players usually show a great passion to them. A special setting is that the products can be sent to in-game friends. This strategy also brings a lot of profits and popularity to Tencent.

In short, the innovative strategies of Honor of Kings continuously bring players fresh experience, which makes it popular on the market.

\section{Discussion}

The four main points of marketing strategies aim at different functions and follow a sequential order. Product marketing introduces the game to the public and leaves a good impression at the first stage. Entertainment marketing then accumulates popularity and opens the market quickly. After the game enters the mature stage, cross-border cooperation can be used to provide a feeling of freshness and help the company generate more profit. A good IP could extend the life of the game and maintain its competitive advantage on the market.

For Honor of Kings, it is now in a mature stage. Thus, the center of marketing should be cross-border cooperation and IP creation. Tencent has a good performance in these aspects. However, it is not easy for the company to bring out something novel which can make an impression. The author's suggestion is that virtual products of different themes will still be attractive. Moreover, the company can enrich the background stories of the characters in the game. The company can create relative animations. For the characters which are originated from historical celebrities, the company can promote their deeds and guide teenagers positively. As for cross-border cooperation, Tencent is expected to corporate with some fashion brands, since both are popular among young people.

\section{Conclusion and Future Work}

This paper delves into the marketing strategies of Tencent Games. The four main points are product marketing, entertainment marketing, cross-border cooperation and intellectual property creation. The classification does not mean these four aspects are overlapped. Instead, they are from different entry points.

Several points can be concluded from the marketing strategies of Tencent Games. First, the innovation team has been involved in the whole process of planning, testing and launch. Therefore, the team can successful catch the highlights and know what may be welcomed. Second, different strategies are used in different periods. A game, as well as its marketing, should follow up with the trend of the times and bring freshness continuously. Furthermore, the company should take on the board users' thoughts and be bold to cooperate with other companies. These points can also be applied to other fields.

However, the research has some flaws. Firstly, it lacks the literature from other countries. This may be because Tencent is a Chinese company and is mainly noticed by Chinese. Second, not so much data related to marketing effect can be found, since it is hard to anticipate what would happen if the strategies were not used.

In the next step, the author will focus on the new promotion tools of Tencent Games. Some questionnaires will be designed to investigate how the people look upon these strategies and how the strategies influence people's behavior. Additionally, more data will be collected to visualize the effectiveness of marketing strategies. If possible, some cases of other companies will be picked out and comparative analysis will be conducted.

\section{Acknowledgment}

The author would like to thank to Stephen Coggeshall of USC for helpful discussions on topic related to this work and enlightenment on market field. Further, the author would like to thank the consultant of Hongtao Jiang of Columbia University, who contributed brilliant ideas on the structure of this paper.

\section{References}

1. Thomala, L. L. Tencent annual online games revenue 2010-2019. 2020 [Online] Available, Retrieved on June 27, 2020, from https://www.statista.com/statistics/527280/tencentannual-online-games-revenue/.

2. Li, T. J. Marketing Strategy Analysis of Mobile Games - Taking Honor of Kings As An Example. Henan Education, 2018-2, 125-129. 
3. Ma, M. Y. Research on marketing strategies of mobile game brands for young consumer groups. Computer Products and Circulation, 2019-2, 161.

4. Qiao, Y. X. Innovative Marketing Strategies and Improvement Approaches in Mobile Online GamesTaking Honor of Kings As An Example. Communication and Copyright, 2018-1, 132-135.

5. Zhang, J., \& Tan, W. Q. Research on Brand Communication Strategy of Online Games-Taking Honor of Kings As An Example. Journal of Nanchang Normal University (Social Sciences), 2019, 40-1, 107-113.

6. Zou, B. Y., \& Chen, X. J. Mobile Game Marketing Strategy Analysis. New Marketing, 2020-4, 67.

7. TGDC-How creativity and design help build content [Online] Available, Retrieved on June 27, 2020, from Tencent Institute of Games website: https://gameinstitute.qq.com/course/detail/10134.

8. Fogel, S. Tencent's 'Honor of Kings' HighestGrossing Mobile Game of 2018 (Analyst). 2019, [Online] Available, Retrieved on June 27, 2020, from https://variety.com/2019/gaming/news/honor-ofkings-highest-grossing-mobile-game-20181203105489/.

9. Chen, C. An exemplary cross-industry collaboration example in China: What do lipstick and China's most popular mobile game have in common? 2019, Retrieved on June 27, 2020, from https://daxueconsulting.com/cross-industrycollaboration-example-china/. 